Justyna Torbiarczyk', Patryk Sobczak', Katarzyna Torbiarczyk', Joanna Miłkowska-Dymanowska', Adam Antczak², Paweł Górski', Adam J. Białas', Wojciech J. Piotrowski'

'Department of Pneumology and Allergy, $1^{\text {st }}$ Chair of Internal Medicine, Medical University of Lodz, Poland

${ }^{2}$ Department of General and Oncological Pulmonology, Medical University of Lodz, Poland

\title{
Is bronchoscopy always justified in diagnosis of haemoptysis? Response to the letter of Tamura et al.
}

\section{Dear Professor Satoh and Co-authors,}

Thank you very much for your interest in our recent study on the use of bronchoscopy for evaluation of bleeding. In order to resolve all doubts, please let us respond point by point:

1. We would like to confirm, that patients with active bleeding were not different concerning the age from non-actively bleeding patients (patients who reported bleeding, but without visible bleeding sites in bronchoscopic examination). The age of 59 is not a cut-off, but median age for both groups (patients with active bleeding and without visible, active bleeding).

2. We used the term "no visible abnormalities" to describe patients which bronchial tree visualized during FOB appeared to be completely normal in terms of anatomy, bronchial mucosa, secretions etc. Therefore, these patients not only had no signs of bleeding but also no signs of any bronchial pathology in FOB. We confirm, that patients with "no visible abnormalities" were younger, comparing to patients with any abnormal signs. This observation seems logic to us, as younger patients have lower risk of plethora of respiratory diseases, i.e. chronic bronchitis. Contrary to your suggestion, we do not provide any data showing the higher occurrence of pathology with high frequency of bleeding in younger patients. We suppose, this may be just the opposite.

3. The location of bleeding was not specifically addressed in our study, due to low number of patients with active bleeding.

We are aware of several limitations of our study. All the more, we are very grateful for initiation of discussion on the role of bronchoscopy for evaluation of bleeding from respiratory tract. We would like to state clearly, that we do not neglect the role of bronchoscopy in this important indication. However, prospective studies are needed to define these indications in specific clinical situations.

\section{Conflict of interest}

The authors declare no conflict of interest. 